\title{
EFFECT OF CRYOGENIC TREATMENT ON THE RHEOLOGICAL PROPERTIES OF GELATIN HYDROGELS
}

S. Van Vlierberghe, P. Dubruel, E. Schacht ${ }^{*}$

Polymer Chemistry \& Biomaterials Research Group, Ghent University, Ghent, Belgium

*Corresponding author: Etienne Schacht

Krijgslaan 281 (Building S4 Bis)

B-9000 Ghent

e-mail: Etienne.Schacht@UGent.be

tel: 003292644497

fax: 003292644972

Keywords: gelatin, cryogenic treatment, freeze-thaw, rheology, mechanical properties 


\section{Abstract}

Above the critical gelation concentration, gelatin has the ability to form a gel when cooled below the sol-gel temperature. In the present work, freeze-thaw cycles were implemented on gelatin solutions possessing varying concentrations. The rheological properties of the cryogels developed were compared with hydrogels formed at room temperature. The results indicated that the rheological properties of cryogels were superior to those of the corresponding hydrogels formed at room temperature. In addition, the critical gelation concentration decreased after (repeated) cryo-treatment(s). The effect of applying various (cryo)parameters, including the number of freeze-thaw cycles, the cooling rate, the thawing rate and the gelatin concentration, on the final material properties was examined. The rheological properties of the cryogels improved with an increasing number of cryo-cycles and a decreasing cooling and thawing rate. In a second part of the work, methacrylamide-modified gelatin was treated cryogenically, followed by in situ UV-irradiation to enable radical crosslinking. It was shown that the crosslinking efficiency improved upon freeze-thawing. The latter was however limited by the gelatin concentration, which affects the chain mobility required to induce phase separation. The present work demonstrates that cryogelation offers possibilities to finetune the mechanical properties of hydrogels. The observed findings are of relevance for the field of tissue engineering, since this concept was previously applied for the development of porous gelatin hydrogels as biomaterials. In addition, a cryogenic treatment can be applied to a wide range of synthetic and natural polymers. 


\section{Introduction}

Cryotropic gelation is a specific type of gelation taking place upon cryogenic treatment of gelforming systems. A requirement for the processes resulting in the formation of cryogels, is crystallisation of the bulk of the low-molecular-weight liquid present in the initial system.(1, 2) Due to the crystallisation of the pure solvent, the total volume of the non-frozen liquid microphase (NFLMP) is lower than the initial reaction volume. As a result, the polymer and/or monomer concentration in the NFLMP is higher than the initial concentration. The polymer gel phase is formed during one of the stages of the cryogenic treatment: during freezing of the initial system, during storage of the samples in the frozen state or during thawing of the frozen specimens.(1, 3-6)

Recently, the structuring of different polymers by applying a cryogenic treatment has attracted a lot of attention, as indicated by the following examples. For a thiol-containing poly(acryl amide) derivative, the conditions were optimized in order to obtain an insoluble gel after freezing-thawing. The cryogel retained the shape of the cryo-mould, in contrast to the polymer solution incubated at room temperature.(7) The reaction rate and yield to transform macromolecular thiols into the corresponding disulphides were increased by freeze-thawing of aqueous thiol-containing polymer solutions in the presence of oxidants.(8)

Cryo-induced association and aggregation of $\beta$-glucans resulted in the formation of soft or hard matter depending on the amount of applied freeze-thaw cycles.(9)

Multiple freezing cycles influenced the structure of poly(vinyl alcohol) (PVA) hydrogels in such a way that secondary crystallites were superimposed on primary crystallites which were formed after one temperature cycle. This resulted in materials possessing superior mechanical properties.(10) 
Cryogels possess a broad application field, as illustrated below.(11) Macroporous PVA cryogels were proposed as cell, drug and protein carriers.(12, 13) Agarose cryogel sponges were evaluated as scaffolds for culturing both isolated pancreatic islets and insulinoma cells. $(14,15)$ Porous gelatin and chondroitin sulphate cryogels were selected as carriers for the culturing of a large variety of human cells (e.g. endothelial cells).(16-18) Blends of poly(vinyl alcohol) with different biological macromolecules, such as hyaluronic acid, dextran and gelatin were applied to produce bioartificial hydrogels functioning as potential tissue engineering scaffolds.(19) Macroporous gels, based on agarose, poly(acrylamide) or polymethacrylates were patented as separation media for chromatography.(20) Poly(vinyl alcohol) cryogels were proposed as temperature sensors, making them applicable for monitoring the temperature distribution induced by a heat source used for hyperthermic therapy.(21)

In the present work, a cryogenic treatment is applied on gelatin for biomedical use. Gelatin has been widely applied in medicine as a wound dressing (22) and as an adhesive and absorbent pad for surgical use.(23) In addition, studies on gelatin-based sponges have indicated their potential in the field of tissue engineering.(24-27) In a previous paper, gelatin hydrogels were treated cryogenically to induce porosity, enabling cell ingrowth and diffusion of nutrients towards the cells and drainage of waste products from the matrix.(16, 18) The present study aims an in depth rheological investigation of the influence of freeze-thaw cycles, by evaluating possible effects of the number of cryogenic cycles, the cooling rate and the thawing rate on the mechanical properties of the resulting materials. To distinguish between physical and chemical crosslinking phenomena, the cryogelation of gelatin and methacrylamide modified gelatin will be compared. 


\section{Materials and Methods}

\subsection{Materials}

Gelatin (type B), isolated from bovine skin by an alkaline process, was kindly supplied by Rousselot, Ghent, Belgium. Gelatin samples possessing an iso-electric point of 5 and a Bloom strength of 257 were used. Methacrylic anhydride (MAA) was purchased from Aldrich (Bornem, Belgium) and was used as received. Dialysis membranes Spectra/Por ${ }^{\circledR} 4$ (MWCO 12,000-14,000 Da) were obtained from Polylab (Antwerp, Belgium). 1-[4-(2Hydroxyethoxy)-phenyl]-2-hydroxy-2-methyl-1-propane-1-one (Irgacure ${ }^{\circledR}$ 2959) was a kind gift from Ciba Speciality Chemicals N.V. (Groot-Bijgaarden, Belgium).

\subsection{Development of cryogels}

\subsubsection{Cryogenic treatment of gelatin}

Aqueous gelatin solutions possessing varying concentrations $(0.2-2 \mathrm{w} / \mathrm{v} \%)$ were prepared by adding double-distilled water to gelatin at $40^{\circ} \mathrm{C}$. Next, the solutions were cooled to room temperature and subjected to a cryogenic treatment. One freeze-thaw cycle included an incubation step at $-30^{\circ} \mathrm{C}$ during 24 hours, followed by a 24 -hour incubation period at $5^{\circ} \mathrm{C}$. As controls, non-frozen hydrogels were stored at $5^{\circ} \mathrm{C}$ for the same incubation time.

For the cryogels developed, possible effects of the cryogenic parameters applied (cooling rate, freezing temperature, thawing rate) were also evaluated.

The cooling rate and freezing temperature were programmed using a Julabo, type FP40-ME (Julabo, Seelbach, Germany).(18) In order to evaluate the influence of the cooling rate on the cryogels developed, all materials were cooled from $21^{\circ} \mathrm{C}$ to $-20^{\circ} \mathrm{C}$ at a certain speed $(0.1$ - 
$1^{\circ} \mathrm{C} / \mathrm{min}$ ). Next, the samples were heated to $5^{\circ} \mathrm{C}$ at a thawing rate of $0.5^{\circ} \mathrm{C} / \mathrm{min}$. Finally, the scaffolds were incubated overnight at $5^{\circ} \mathrm{C}$.

In a subsequent part of the present work, the influence of the thawing rate was evaluated by freezing the gels for 30 minutes at $-20^{\circ} \mathrm{C}$. Next, the cryogels were thawed to $2^{\circ} \mathrm{C}$ at various thawing rates $\left(0.06-1^{\circ} \mathrm{C} / \mathrm{min}\right)$ followed by an overnight incubation at $5^{\circ} \mathrm{C}$.

\subsubsection{Synthesis and characterization of methacrylamide-modified gelatin}

After swelling of $100 \mathrm{~g}$ gelatin (35 mmol $\varepsilon$-amine-sidegroups of lysine and hydroxylysine) in $1 \mathrm{l}$ phosphate buffer ( $\mathrm{pH} 7.8$ ) for one hour, the solution was heated to $40^{\circ} \mathrm{C}$. When a homogeneous gelatin solution was obtained, one equivalent of methacrylic anhydride (5.66 $\mathrm{ml}, 0.038 \mathrm{~mol}$ ) was added. The reaction mixture was stirred vigorously for one hour at $40^{\circ} \mathrm{C}$. Next, the mixture was diluted with $1 \mathrm{l}$ double distilled water and transferred in dialysis membranes (Spectra/Por® 4, MWCO 12,000-14,000 Da). After one day of dialysis in water at $40^{\circ} \mathrm{C}$, the obtained derivative was freeze-dried.

${ }^{1} \mathrm{H}$ NMR-spectra of modified gelatin were recorded at $40^{\circ} \mathrm{C}$ in deuterated water (Figure 1). The degree of substitution was obtained after comparison of the integrations of the characteristic peaks of the methacrylamide-moieties (i.e. $I_{5.62}$ ppm and $I_{5.85}$ ppm) and the integration of a peak of amino acids, which are not involved in the modification (e.g. Val + Leu + Ile at $1.12 \mathrm{ppm})$. Based on the known amino acid composition of the gelatin applied, the degree of substitution can be calculated, as indicated by the following equation:

$$
\text { DS }(\%)=0.3836 \mathrm{~mol} \times\left(\mathrm{I}_{5.7 \mathrm{ppm}} / \mathrm{I}_{1.1 \mathrm{ppm}}\right) \times(100 / 0.0385 \mathrm{~mol})
$$

Gelatin was chemically modified with methacrylamide side groups, resulting in a degree of substitution (DS) of 60\%.(28) 
2.2.3. Cryogenic treatment of methacrylamide-modified gelatin

To an aqueous solution of gelatine-methacrylamide (gel-MOD), 2 mol\% photo-initiator Irgacure ${ }^{\circledR}$ 2959, as calculated to the methacrylamide side chains, was added. Gels were UVcured in situ, during rheology, using a Novacure 2100 spot curing system (EXFO Photonic Solutions Inc.).

\subsection{Rheological evaluation of cryogels}

The rheological properties of the gels were evaluated using a rheometer type Physica MCR301 (Anton Paar, Sint-Martens-Latem, Belgium). Oscillation measurements were performed using two parallel plates, with the upper plate having a diameter of $50 \mathrm{~mm}$. Amplitude scans were performed at $5^{\circ} \mathrm{C}$, at a frequency of $1 \mathrm{~Hz}$ and with a gap of $0.5 \mathrm{~mm}$. Mechanical spectra were obtained at $5^{\circ} \mathrm{C}$, using a strain of $0.5 \%$ and a gap of $0.5 \mathrm{~mm}$. G' and G'’ were measured using oscillation rheology.

\subsection{Statistical analysis}

Statistical analysis was performed on the data, using the student $t$-test. Two values were considered to be significantly different when $p<0.05$. 


\section{Results and Discussion}

In the present paper, an in depth analysis of the cryogelation of gelatin is presented. Since porous materials possess widespread applications as separation media, biomaterials, etc., a profound understanding of the cryogenic parameters affecting the final material properties is of great importance. In addition to a possible effect of the gelatin concentration, we also studied possible influences of the number of cryogenic cycles, the cooling rate and the thawing rate on the rheological properties of the resulting cryogels. To distinguish between physical and chemical crosslinking phenomena, both the cryogelation of gelatin and methacrylamide-modified gelatine (gel-MOD) were evaluated.

\subsection{Cryogenic treatment of gelatin}

\subsubsection{Effect of the gelatin concentration}

In a first part of the work, the influence of the gelatin concentration on the rheological properties of gelatin hydrogels and cryogels was compared. Since knowledge of the linear visco-elastic range of a material is required prior to performing rheological measurements, an amplitude scan of the different gels was performed first (data not shown). A strain of $0.5 \%$ was selected for all further measurements since this was the highest value within the linear visco-elastic area. Next, the mechanical spectra of the gels were recorded. These tests showed a limited frequency-dependence of the storage modulus (data not shown). The storage modulus gives an estimation of the strain energy which is reversibly stored in a substance. Since it is recoverable, it thus characterizes the elastic behaviour of a sample.

The influence of the polymer concentration on the cryogenic treatment was studied by applying one freeze-thaw cycle on various gelatin concentrations (0.2 - $2 \mathrm{w} / \mathrm{v} \%)$. These 
cryogels were compared with unfrozen hydrogels possessing similar polymer concentrations and incubation periods. Figure 2 shows the storage moduli corresponding with various gelatin concentrations. The results indicate that the storage moduli (G') of the cryogels (after a single freezing cycle) were higher compared to the non-frozen hydrogels irrespective of the applied gelatin concentration $(0.2-2 \mathrm{w} / \mathrm{v} \%)$. In addition, the ratio of the storage moduli of the cryogels and the hydrogels (i.e. G’(Cryogel)/G'(Hydrogel); see Figure 2, dotted line) revealed a maximum at a gelatin concentration of $0.5 \mathrm{w} / \mathrm{v} \%$. This corresponds with a "cryogel" in the "gel” state and a "hydrogel” in the "sol” state (Figure 3). The storage and loss moduli of a 0.5 w/v\% hydrogel are very low (as indicated by the arrow in Figure 3), while G' of the cryogel is three times higher than G'”. This indicates that a cryogenic treatment leads to gel formation at gelatin concentrations lower than the critical gelation concentration at room temperature. It should be noted that the observed decrease in gelation concentration is only apparent, since the polymer chains are accumulated in the gelation region (i.e. non-frozen liquid microphase), resulting in a local increase of the gelatin concentration. This effect has been observed for any type of cryotropic gelation.(1) The structuring of gelatin upon cryogenic treatment is very similar to the freeze-thaw behaviour of cereal $\beta$-glucans and xanthan. $(3,9)$ The gelatin gels, obtained after the cryogenic treatment, also belong to the category of physically crosslinked gels whose three-dimensional structure is mainly stabilized by multiple hydrogen bonds and hydrophobic interactions in the junction zones of the polymeric network.(9)

\subsubsection{Effect of the number of cryogenic cycles}

In addition to the gelatin concentration, we also investigated the effect of the number of cryogenic cycles applied on the rheological properties of the obtained cryogels. For these measurements, $0.5 \mathrm{w} / \mathrm{v} \%$ and $2 \mathrm{w} / \mathrm{v} \%$ gelatin hydrogels were selected. In Figure 4, the storage moduli are plotted as a function of the gelatin concentration and the number of freeze- 
thaw cycles. The results indicate that the rheological properties of cryogels can be improved by increasing the number of cryogenic cycles. This trend was observed for both gelatin concentrations (i.e. 0.5 and 2 w/v\%) studied. The latter can be explained by the formation of additional crosslinks during repeated freezing/thawing cycles. The physical gelation of gelatin is a dynamic process in which bonds are broken and formed continuously. With an increasing incubation time, a more stable conformation is obtained. However, due to physical limitations, equilibrium is never reached. The latter phenomenon is called "frustrated renaturation”.(29, 30) With the parameters applied in the present work, a kinetic competition exists between the self-gelling process below the sol-gel temperature $\left( \pm 30^{\circ} \mathrm{C}\right)$ and the freezing process below $0^{\circ} \mathrm{C}$. There are thus two possible explanations for the improved mechanical properties of cryo-treated gelatin gels. A first possibility is that the freezing rate is faster than the rate of physical gelation. This implies that cryotropic gelation could occur on gelatin in the sol-state. A second possibility is that physical gelation takes place faster than cryotropic gelation. Enough chain mobility apparently remains within the hydrogel in order to enable further chain entanglement resulting in a gel possessing superior rheological properties. Kinetics of the freezing and the self-gelling process could also be comparable, however distinguishing both phenomena is very difficult, if not impossible. A progressive increase in mechanical properties after multiple freezing/thawing cycles has been reported for xanthan and poly(vinyl alcohol) cryogels.(3, 6) This was ascribed to the formation of microcrystalline zones acting as junction knots in the supramolecular PVA network. The dimensions of the micro crystallites formed were influenced by the number of applied freezing/thawing cycles.(1, 31)

The observed variations in mechanical properties can affect the degradation behaviour of the gelatin scaffolds developed earlier.(18) It can be anticipated that increased mechanical properties, related to a more efficient physical crosslinking (i.e. triple helix formation), would 
adversely affect the degradation rate. This is of relevance in the field of tissue engineering, since, ideally, the degradation time of the scaffolds should match the time required for new tissue formation.

\subsubsection{Influence of the cooling rate during the freezing step}

Experiments using PVA based cryogels have indicated that over a wide range of cooling rates $\left(0.1^{\circ} \mathrm{C} / \mathrm{min}\right.$ to $\left.17^{\circ} \mathrm{C} / \mathrm{min}\right)$, the effect on the physical characteristics of the gels was relatively low.(32) However, for blends of amylopectin and amylose, the effect of the cooling rate showed a more pronounced influence on the material properties.(5) In that study, hydrogel samples were incubated at various temperatures for similar incubation times and thawed subsequently at the same rate. The cryogels developed, could be distinguished by their appearance and their swelling properties. However, the observed differences were attributed to the storage in the frozen state, despite the fact that a different cooling rate was applied during the treatment.

For gelatin, we anticipated that the cooling rate would influence the rheological properties since gelation occurs via triple helix formation. These helices act as junction zones within the network.(30) A faster cooling process could hinder the triple helix formation, and thus the degree of physical gelation. Figure 5 indicates that for $1 \mathrm{w} / \mathrm{v} \%$ hydrogels, the storage modulus decreased with an increasing cooling rate. A higher freezing rate thus indeed disturbs the formation of chain entanglements and thus the physical gelation. A similar trend was observed for $10 \mathrm{w} / \mathrm{v} \%$ hydrogels (data not shown) indicating that within the concentration range investigated, the gelatin concentration does not affect the kinetic effects observed at low concentrations. 


\subsubsection{Effect of the thawing rate}

The above results clearly indicate that the conditions applied for the development of cryogels, strongly affect the final material mechanical properties. For the effect of the thawing rate, it has been shown that the influence depends on the type of hydrogel crosslinks.(1) For cryogels crosslinked by covalent or ionic bonds, the thawing conditions of specimens usually have a limited influence on the final material properties. However, for cryogels in which hydrogen bond formation is the predominant crosslinking mechanism, the resulting gels often are fairly sensitive to the thawing conditions applied.(1) In the present work, the thawing rate was varied from 0.06 to $1^{\circ} \mathrm{C} / \mathrm{min}$. The results of the rheological studies (Figure 6), indicated a decrease in G' with an increasing thawing rate. This trend, observed earlier for PVA, is typical for thermally reversible cryogels in which hydrogen bonding is the predominant type of intermolecular junctions stabilising the polymer network.(1, 6, 32)

\subsection{Cryogenic treatment of methacrylamide-modified gelatin}

The above results indicate that the cryogenic treatment enhances the triple helix formation of gelatin chains. In addition to this effect, an influence on the subsequent chemical crosslinking step was also anticipated. The latter is affected by the separation between the different methacrylamide moieties as well as their mobility. In Figure 7, storage moduli before and after UV-treatment, for a $10 \mathrm{w} / \mathrm{v} \%$ non-frozen gelatin hydrogel were compared with those from a cryogel, developed by applying one freeze-thaw cycle. In accordance with nonmodified gels, G' (cryogel) before UV-irradiation was higher than that G'(hydrogel). However, the increase in storage modulus during the chemical crosslinking was also higher 
for the cryogel compared to the non-frozen hydrogel. The latter is a first indication that freeze-thawing has a favourable effect on the crosslink efficiency of $10 \mathrm{w} / \mathrm{v} \%$ methacrylamide-modified gelatin. During the cryo-treatment, phase separation occurs which probably reduces the distance between neighbouring vinyl bonds and thus facilitates the chemical crosslinking. On the other hand, a change in gelatin concentration alters the mobility of the polymer chains. Therefore, $15 \mathrm{w} / \mathrm{v} \%$ gelatin hydrogels were also freeze-thawed and their rheological properties before and after UV-curing were compared with non-frozen hydrogels (Figure 8). Before chemical crosslinking, G' of both hydrogel types were in the same range. This is probably due to a lack of chain mobility below the sol-gel temperature, which hinders phase separation below the solvent crystallisation temperature, thus avoiding an increase in the polymer concentration within the "non-frozen liquid microphase". After UV-treatment, the storage modulus of the hydrogel is even higher than that of the cryogel, which can be explained by a possible phase separation of the initiator molecules and the methacrylamide moieties related to the cryo-treatment.

The observed findings are of importance in the field of tissue engineering since the variation of the applied cryogenic parameters affects the mechanical properties of the resulting materials. The latter influences the degradation profile of the (cell seeded) scaffolds which, ideally, should match the rate of new tissue formation. Without any doubt, this process can and will be applied on a large number of (natural) polymers. 


\section{Conclusions}

Gelatin cryogels, developed by freeze-thaw treatments, possess a lower critical gelation concentration compared to hydrogels formed at room temperature. This implies that lower gelatin concentrations can be applied for developing gels possessing similar rheological properties. This concept was already successfully applied for a variety of polymers. The present work however demonstrates that the self-gelling properties of gelatin (below the solgel temperature) do not necessarily limit certain processing possibilities. In addition, applying a freeze-thaw cycle to methacrylamide-modified gelatin enhances the subsequent crosslink efficiency of the resulting materials significantly. Cryogelation of materials can reduce the required amount of material to a great extent, which benefits not only the cost, but also offers the possibility of decreasing the amount of added initiator.

The present work also indicates that the concept is limited to gelatin and methacrylamidemodified gelatin concentrations for which the chain mobility below the sol-gel temperature is sufficient to enable phase separation and subsequent cryo-concentration within the non-frozen liquid microphase. 


\section{Acknowledgements}

The authors would like to acknowledge the Research Foundation - Flanders (FWO) for the research grant assigned to S. Van Vlierberghe. 


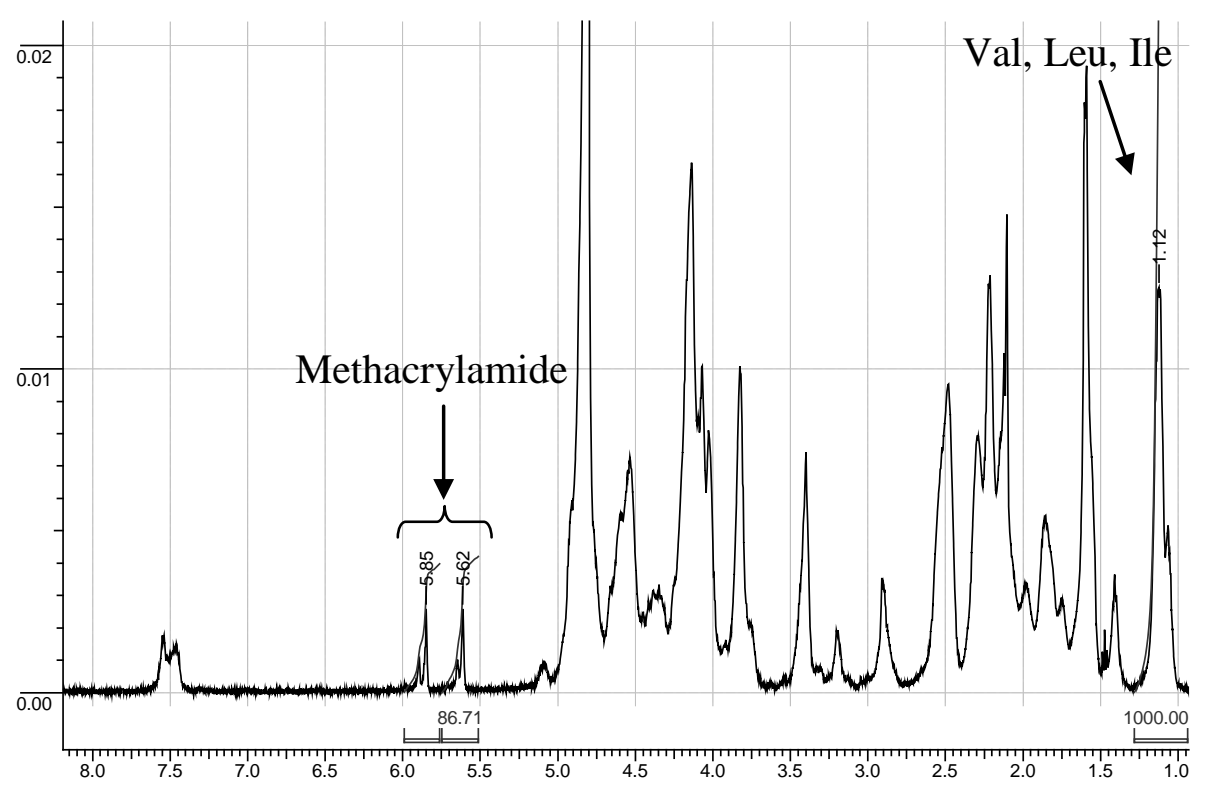

Figure 1

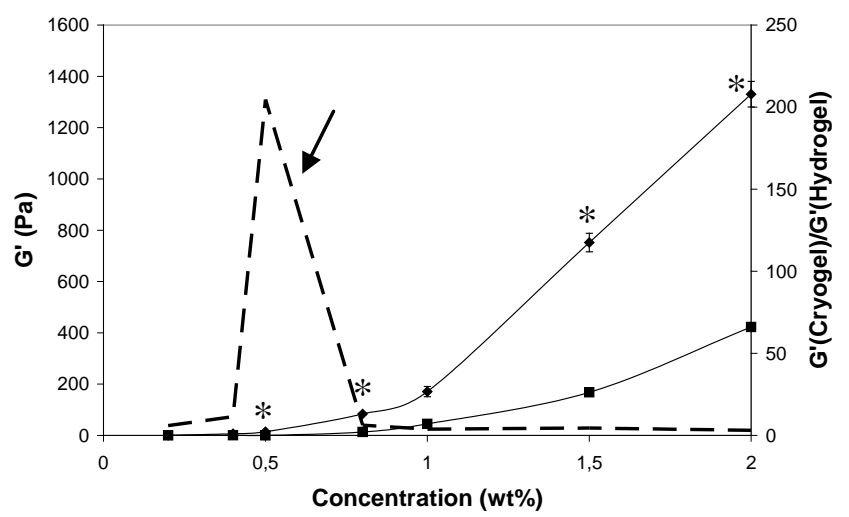

Figure 2

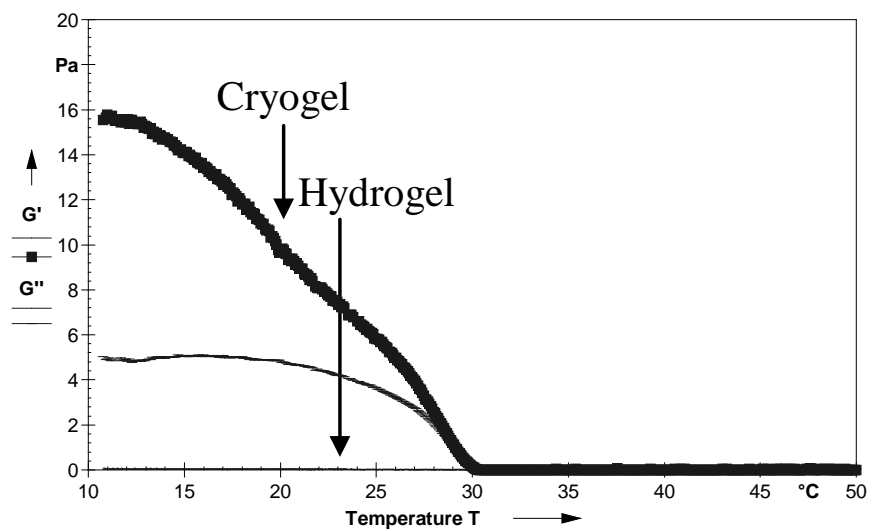

Figure 3 


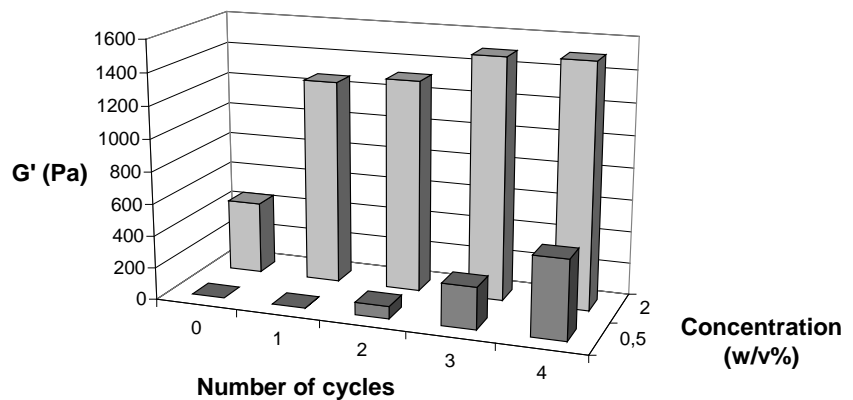

Figure 4

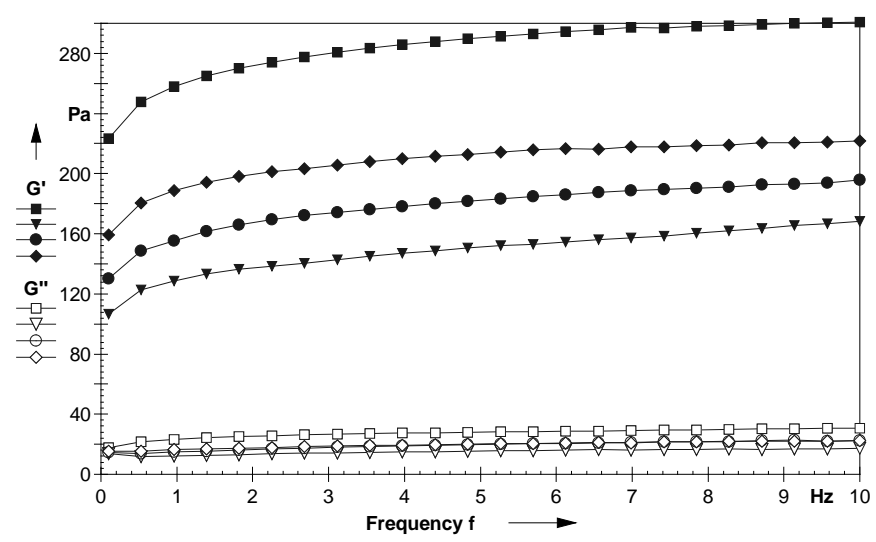

Figure 5

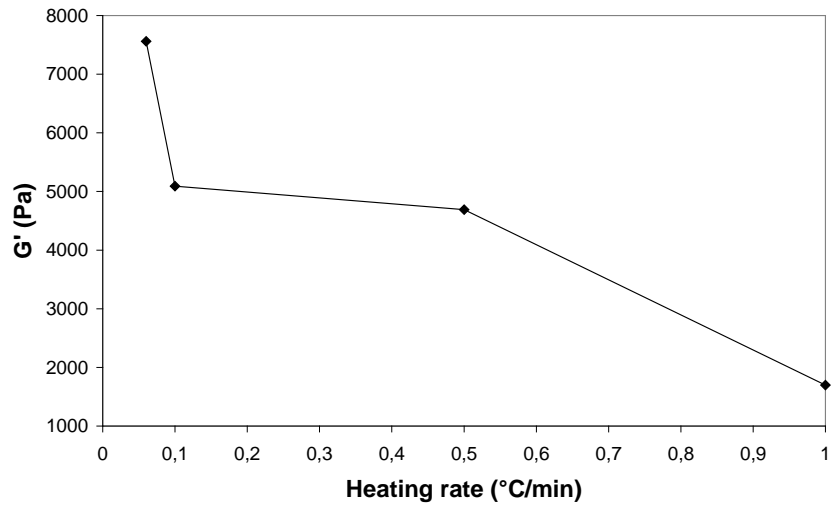

Figure 6 


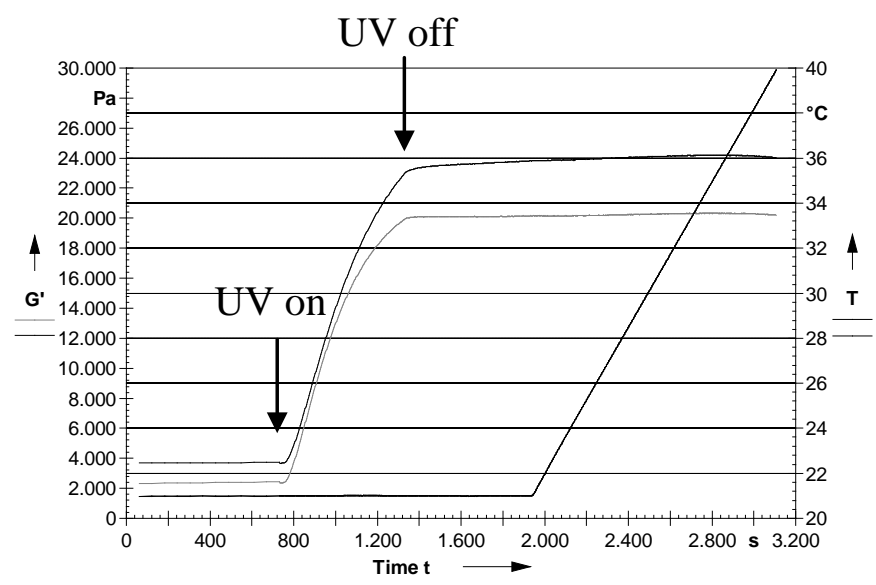

Figure 7

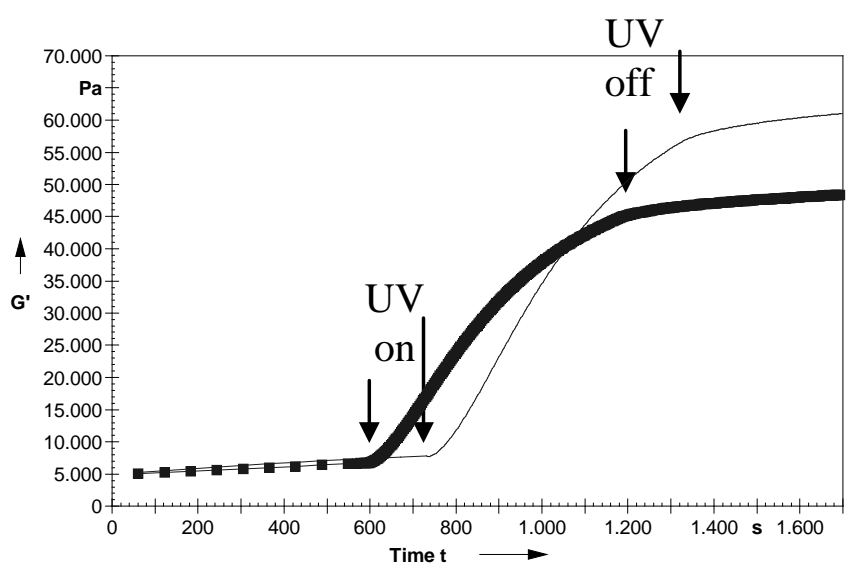

Figure 8 
Figure 1. $\quad{ }^{1} \mathrm{H}-\mathrm{NMR}$ spectrum of gel-MOD recorded in $\mathrm{D}_{2} \mathrm{O}$ at $40^{\circ} \mathrm{C}$.

Figure 2. Influence of gelatin concentration (w/v\%) on $\mathrm{G}^{\prime}\left(0.5 \%\right.$ strain, $0.85 \mathrm{~Hz}, 5^{\circ} \mathrm{C}$, gap $0.5 \mathrm{~mm}$ ) for unfrozen gelatin solutions ( $)$ and for gelatin cryogels formed by a single cycle of freezing to $-30^{\circ} \mathrm{C}(\diamond)\left({ }^{*} P<0.05\right)$.

Figure 3. Temperature scans $\left(0.5 \%\right.$ strain, $\left.0.85 \mathrm{~Hz}, \mathrm{~F}_{\mathrm{N}} 0 \mathrm{~N}\right)$ of unfrozen $0.5 \mathrm{w} / \mathrm{v} \%$ gelatin solutions (hydrogel) and after applying one freeze-thaw cycle (cryogel).

Figure 4. Effect of the number of freeze-thaw cycles on $\mathrm{G}^{\prime}\left(0.5 \%\right.$ strain, $0.85 \mathrm{~Hz}, 5^{\circ} \mathrm{C}$, gap $0.5 \mathrm{~mm}$ ) for different gelatin concentrations.

Figure 5. Mechanical spectra $\left(0.5 \%\right.$ strain, $5^{\circ} \mathrm{C}$, gap $\left.0.5 \mathrm{~mm}\right)$ showing the influence of the cooling rate on G' (filled symbols) and G', (open symbols) of a $1 \mathrm{w} / \mathrm{v} \%$ gelatin solution. The applied cooling rates were $0.1^{\circ} \mathrm{C} / \mathrm{min}(\mathbf{*}), 0.3^{\circ} \mathrm{C} / \mathrm{min}(\boldsymbol{\diamond})$, $0.5^{\circ} \mathrm{C} / \mathrm{min}(\bullet)$ and $1^{\circ} \mathrm{C} / \mathrm{min}(\boldsymbol{\nabla})$.

Figure 6. Effect of the thawing rate on the storage modulus (G') of $10 \mathrm{w} / \mathrm{v} \%$ hydrogels.

Figure 7. Storage modulus as a function of time for $10 \mathrm{w} / \mathrm{v} \%$ unfrozen methacrylamide gelatin hydrogels (grey) and for cryogels formed by one freeze-thaw cycle (black). The hydrogels were UV-irradiated during 10 minutes, followed by a 10 -minute after-curing step. In a final part, a temperature-scan from $21^{\circ} \mathrm{C}$ to $40^{\circ} \mathrm{C}$ was performed $\left(\mathrm{F}_{\mathrm{N}}=0.2 \mathrm{~N}\right)$.

Figure $8 \quad G^{\prime}$ at different time points of $15 \mathrm{w} / \mathrm{v} \%$ unfrozen hydrogels (-) and of cryogels after applying one cryo-treatment ( $\mathbf{(})$. The gels were in situ UV-cured during 10 minutes at $5^{\circ} \mathrm{C}\left(\right.$ gap $\left.1 \mathrm{~mm}, \mathrm{~F}_{\mathrm{N}}=0.2 \mathrm{~N}\right)$. 


\section{References}

1. Lozinsky, V.I. (2002). Cryogels on the basis of natural and synthetic polymers: Preparation, properties and application, Uspekhi Khimii, 71(6): 559-585.

2. Kirsebom, H., Aguilar, M.R., San Roman, J., Fernandez, M., Prieto, M.A. and Bondar, B. (2007). Macroporous Scaffolds Based on Chitosan and Bioactive Molecules\{dagger\}, Journal of Bioactive and Compatible Polymers, 22(6): 621-636.

3. Giannouli, P. and Morris, E.R. (2003). Cryogelation of xanthan, Food Hydrocolloids, 17(4): 495-501.

4. Podorozhko, E.A., Kurskaya, E.A., Kulakova, V.K. and Lozinsky, V.I. (2000). Cryotropic structuring of aqueous dispersions of fibrous collagen: influence of the initial $\mathrm{pH}$ values, Food Hydrocolloids, 14(2): 111-120.

5. Lozinsky, V.I., Damshkaln, L.G., Brown, R. and Norton, I.T. (2000). Study of cryostructuration of polymer systems. XVIII. Freeze-thaw influence on water-solubilized artificial mixtures of amylopectin and amylose, Journal of Applied Polymer Science, 78(2): $371-381$.

6. Lozinsky, V.I. and Damshkaln, L.G. (2000). Study of cryostructuration of polymer systems. XVII. Poly(vinyl alcohol) cryogels: Dynamics of the cryotropic gel formation, Journal of Applied Polymer Science, 77(9): 2017-2023.

7. Vainerman, E.S., Lozinsky, V.I. and Rogozhin, S.V. (1981). Study of Cryostructurization of Polymer Systems .I. Structure Formation in Solutions of ThiolContaining Polymers under Freezing-Thawing, Colloid and Polymer Science, 259(12): 11981201. 
8. Lozinsky, V.I., Golovina, T.O. and Gusev, D.G. (2000). Study of cryostructuration of polymer systems: XIII. Some characteristic features of the behaviour of macromolecular thiols in frozen aqueous solutions, Polymer, 41(1): 35-47.

9. Lazaridou, A. and Biliaderis, C.G. (2004). Cryogelation of cereal beta-glucans: structure and molecular size effects, Food Hydrocolloids, 18(6): 933-947.

10. Willcox, P.J., Howie, D.W., Schmidt-Rohr, K., Hoagland, D.A., Gido, S.P., Pudjijanto, S., Kleiner, L.W. and Venkatraman, S. (1999). Microstructure of poly(vinyl alcohol) hydrogels produced by freeze/thaw cycling, Journal of Polymer Science Part BPolymer Physics, 37(24): 3438-3454.

11. Lozinsky, V.I., Galaev, I.Y., Plieva, F.M., Savinal, I.N., Jungvid, H. and Mattiasson, B. (2003). Polymeric cryogels as promising materials of biotechnological interest, Trends in Biotechnology, 21(10): 445-451.

12. Lozinsky, V.I., Zubov, A.L. and Titova, E.F. (1997). Poly(vinyl alcohol) cryogels employed as matrices for cell immobilization .2. Entrapped cells resemble porous fillers in their effects on the properties of PVA-cryogel carrier, Enzyme and Microbial Technology, 20(3): 182-190.

13. Veronese, F.M., Mammucari, C., Caliceti, P., Schiavon, O. and Lora, S. (1999). Influence of PEGylation on the Release of Low and High Molecular-Weight Proteins from PVA Matrices, Journal of Bioactive and Compatible Polymers, 14(4): 315-330.

14. Bloch, K., Lozinsky, V.I., Galaev, I.Y., Yavriyanz, K., Vorobeychik, M., Azarov, D., Damshkaln, L.G., Mattiasson, B. and Vardi, P. (2005). Functional activity of insulinoma cells (INS-1E) and pancreatic islets cultured in agarose cryogel sponges, Journal of Biomedical Materials Research Part A, 75A(4): 802-809. 
15. Kawazoe, N., Xiaoting, L., Tateishi, T. and Guoping, C. (2009). Three-dimensional Cultures of Rat Pancreatic RIN-5F Cells in Porous PLGA-collagen Hybrid Scaffolds, Journal of Bioactive and Compatible Polymers, 24(1): 25-42.

16. Dubruel, P., Unger, R., VanVlierberghe, S., Cnudde, V., Jacobs, P.J.S., Schacht, E. and Kirkpatrick, C.J. (2007). Porous Gelatin Hydrogels: 2. In Vitro Cell Interaction Study, Biomacromolecules, 8(2): 338-344.

17. Van Vlierberghe, S., Dubruel, P., Lippens, E., Masschaele, B., Van Hoorebeke, L., Cornelissen, M., Unger, R., Kirkpatrick, C.J. and Schacht, E. (2008). Toward modulating the architecture of hydrogel scaffolds: curtains versus channels, Journal of Materials ScienceMaterials in Medicine, 19(4): 1459-1466.

18. VanVlierberghe, S., Cnudde, V., Dubruel, P., Masschaele, B., Cosijns, A., DePaepe, I., Jacobs, P.J.S., VanHoorebeke, L., Remon, J.P. and Schacht, E. (2007). Porous Gelatin Hydrogels: 1. Cryogenic Formation and Structure Analysis, Biomacromolecules, 8(2): 331337.

19. Cascone, M.G., Lazzeri, L., Sparvoli, E., Scatena, M., Serino, L.P. and Danti, S. (2004). Morphological evaluation of bioartificial hydrogels as potential tissue engineering scaffolds, Journal of Materials Science-Materials in Medicine, 15(12): 1309-1313.

20. Mattiasson and Galaev, inventors; Separation medium, its preparation and its use.

21. Lukas, L.A., Surry, K.J.M. and Peters, T.M. (2001). Temperature dosimetry using MR relaxation characteristics of poly(vinyl alcohol) cryogel (PVA-C), Magnetic Resonance in Medicine, 46(5): 1006-1013.

22. Tucci, M.G., Ricotti, G., Mattioli-Belmonte, M., Gabbanelli, F., Lucarini, G., Orlando, F., Viticchi, C., Bigi, A., Panzavolta, S., Roveri, N., Morganti, G. and Muzzarelli, R.A.A. (2001). Chitosan and Gelatin as Engineered Dressing for Wound Repair, Journal of Bioactive and Compatible Polymers, 16(2): 145-157. 
23. Wang, X., Yan, Y. and Zhang, R. (2006). A Comparison of Chitosan and Collagen Sponges as Hemostatic Dressings, Journal of Bioactive and Compatible Polymers, 21(1): 3954.

24. Yamamoto, M., Tabata, Y. and Ikada, Y. (1999). Growth Factor Release from Gelatin Hydrogel for Tissue Engineering, Journal of Bioactive and Compatible Polymers, 14(6): 474489.

25. Wei, X., Xiaohong, W., Yongnian, Y., Wei, Z., Zhuo, X., Feng, L., Rendong, W. and Renji, Z. (2007). Rapid Prototyping Three-Dimensional Cell/Gelatin/Fibrinogen Constructs for Medical Regeneration, Journal of Bioactive and Compatible Polymers, 22(4): 363-377.

26. Zhang, T., Yan, Y., Wang, X., Xiong, Z., Lin, F., Wu, R. and Zhang, R. (2007). Three-dimensional Gelatin and Gelatin/Hyaluronan Hydrogel Structures for Traumatic Brain Injury, Journal of Bioactive and Compatible Polymers, 22(1): 19-29.

27. Wei, X., Xiaohong, W., Yongnian, Y. and Renji, Z. (2008). A Polyurethane-Gelatin Hybrid Construct for Manufacturing Implantable Bioartificial Livers, Journal of Bioactive and Compatible Polymers, 23(5): 409-422.

28. Van den Bulcke, A.I., Bogdanov, B., De Rooze, N., Schacht, E.H., Cornelissen, M. and Berghmans, H. (2000). Structural and rheological properties of methacrylamide modified gelatin hydrogels, Biomacromolecules, 1(1): 31-38.

29. Djabourov, M. (1988). Architecture of Gelatin Gels, Contemporary Physics, 29(3): 273-297.

30. Tosh, S.M., Marangoni, A.G., Hallett, F.R. and Britt, I.J. (2003). Aging dynamics in gelatin gel microstructure, Food Hydrocolloids, 17(4): 503-513.

31. Hassan, C.M., Ward, J.H. and Peppas, N.A. (2000). Modeling of crystal dissolution of poly(vinyl alcohol) gels produced by freezing/thawing processes, Polymer, 41(18): 67296739. 
32. Lozinsky, V.I. and Plieva, F.M. (1998). Poly(vinyl alcohol) cryogels employed as matrices for cell immobilization. 3. Overview of recent research and developments, Enzyme and Microbial Technology, 23(3-4): 227-242. 